\title{
Appareil
}

$17 \mid 2016$

Art et médium 1 : le médium de l'art

\section{Dans le corps de l'œuvre : pour une préhistoire du médium chez Diderot}

\section{Giuseppe Di Liberti}

\section{OpenEdition}

1 Journals

\section{Édition électronique}

URL : http://journals.openedition.org/appareil/2293

DOI : 10.4000/appareil.2293

ISSN : 2101-0714

Éditeur

MSH Paris Nord

Référence électronique

Giuseppe Di Liberti, « Dans le corps de l'œuvre : pour une préhistoire du médium chez Diderot », Appareil [En ligne], 17 | 2016, mis en ligne le 11 juillet 2016, consulté le 30 juillet 2020. URL : http:// journals.openedition.org/appareil/2293; DOI : https://doi.org/10.4000/appareil.2293

Ce document a été généré automatiquement le 30 juillet 2020.

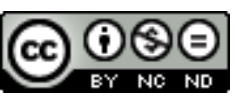

Appareil est mis à disposition selon les termes de la Licence Creative Commons Attribution - Pas d'Utilisation Commerciale - Pas de Modification 4.0 International. 


\title{
Dans le corps de l'œuvre : pour une préhistoire du médium chez Diderot
}

\author{
Giuseppe Di Liberti
}

\section{NOTE DE L'AUTEUR}

Je tiens ici à remercier Pascal Krajewski pour avoir encouragé cet article ainsi qu'Agathe Henssien et Pierre Leger pour leurs relectures ponctuelles et attentives.

\section{Le spectateur, le critique, le philosophe}

1 Dans l'incipit du Laocoon, ouvrage qui pourrait à juste titre être considéré comme le point de départ de toute réflexion sur l'intermédialité à l'âge moderne, Lessing présente à son lecteur trois figures emblématiques de ceux que l'on pourrait désigner aujourd'hui comme des « spectateurs informés » s'étant essayés à la comparaison entre poésie et peinture : l'amateur, « homme d'une grande finesse de goût qui sentait que ces deux arts produisaient en lui les mêmes effets »; le philosophe qui «voulut mieux comprendre notre plaisir et découvrit qu'il découlait dans les deux arts d'une seule et même source ", la beauté, montrant ainsi qu'elle possède des règles générales; le critique d'art "réfléchissant sur la valeur et la répartition de ces règles générales, remarqua[nt] que les unes dominent la peinture, les autres la poésie ${ }^{1}$ ».

Dans sa distribution des rôles, Lessing oublie, sans méchanceté pourrait-on dire, Denis Diderot. Il avait pourtant lu la Lettre sur les sourds et muets, souvent reconnue comme le "Laocoon français", dont il avait écrit un compte rendu élogieux dans le Vossische Zeitung en 1751, l'année même de la publication de la Lettre. Ses éloges s'adressaient surtout à la promptitude d'esprit de Diderot :

Qui ne connait les habitudes de Monsieur Diderot ? Il se lance constamment dans les digressions, il saute d'une idée à l'autre, et le dernier mot d'une période lui paraît une transition suffisante. Le nom de Lettre est peut-être une légère excuse pour ce manque 
de liaison. Sa meilleure excuse cependant, c'est que toutes ses digressions sont pleines d'idées neuves et belles. Si seulement tous les écrivains décousus voulaient bien nous dédommager de la sorte ${ }^{2}$ !

Lessing reconnut donc Diderot en tant que critique d'art plutôt que comme philosophe. L'importance du procès intenté par Diderot aux idées de Batteux échappa [à Lessing], et il se borna à des éloges de nature générale qui montrent qu'il appréciait bien l'ouverture et la mobilité d'un esprit qu'il sentait apparenté au sien, mais qu'il ne discernait pas encore la portée de ses réflexions esthétiques ${ }^{3}$.

D'ailleurs, quinze ans plus tard, dans son Laocoon, il n'y aura aucune trace de la lecture de la Lettre. Pourtant Diderot - et il s'agit de l'un des traits extraordinaires de sa réflexion - a été tout à la fois amateur, philosophe et critique. Il a souvent joué ces rôles simultanément dans le même texte, voire dans la même phrase. Il aurait certainement rejeté l'appellation d'amateur («Nos artistes sont fatigués dans leurs ateliers d'une vermine présomptueuse qu'on appelle des amateurs, et cette vermine nuit beaucoup à leurs travaux ${ }^{4} »$ ) en lui préférant peut-être le qualificatif de connaisseur, auquel manquerait cependant la coloration sentimentale qui convient bien à maintes pages des Salons. Si Dieckmann a reconnu que Diderot avait «fait de la critique "en technicien" et "en philosophe" $"$ ", il faut certainement y ajouter la position du spectateur, qui certes vise à pénétrer les secrets de fabrication des objets artistiques et à comprendre les règles générales des arts ainsi que leur rapport à la nature et à la vérité, mais qui juge toujours à partir des effets que l'œuvre suscite en lui.

D'une certaine manière, à ces trois figures correspondent trois sens du médium chez Diderot, tout en sachant que Diderot n'utilise évidemment pas ce terme et que la tripartition arbitraire adoptée ici vise simplement à saisir trois plans qui sont intimement entrelacés. Nous ne souhaitons pas reconstruire ici une sorte de théorie du médium chez Diderot, mais nous cherchons plutôt à déterminer certaines caractéristiques du médium à partir de la réflexion sur les arts de Diderot, considérée comme geste fondateur de la critique d'art liée à la philosophie. Nous nous limiterons à parcourir quelques passages des Salons, donc nous resterons surtout dans le domaine de la peinture, puisqu'elle demeure certainement le domaine exemplaire pour examiner comment le spectateur, le critique et le philosophe travaillent ensemble. Il serait néanmoins envisageable de vérifier si les distinctions proposées ici sont également valables dans les autres domaines artistiques.

Cette tripartition nous semble bien représenter les différentes étapes des analyses d'œuvres par Diderot : commencer par les effets suscités par l'œuvre, puis comprendre comment l'œuvre a "fabriqué » (ou non) de tels effets, enfin inscrire ces analyses dans une interrogation plus large qui concerne aussi bien l'éthique que la relation entre la vérité (de l'œuvre) et la nature. Seule la profonde cohérence de ces trois plans peut conduire le Philosophe à la pleine appréciation d'une œuvre ${ }^{6}$.

\section{Trois voies vers le médium}

En acceptant la proposition de Grimm de rédiger des comptes rendus des Salons pour la Correspondance littéraire, Diderot adopte avant tout la position du spectateur et note régulièrement les effets sentimentaux que les tableaux produisent sur lui. En 1763, il déclare : « Voilà, mon ami, tout ce que j'ai vu au Salon. Je vous l'écris au courant de la plume. [...] J'ai senti, et j'ai dit comme je sentais ${ }^{7}{ }^{\prime}$. Deux ans plus tard, en s'adressant au 
peintre avec ses Essais sur la peinture, dans la partie consacrée à la composition, il exprime très clairement son attente et son désir : "Touche-moi, étonne-moi, déchiremoi, fais-moi tressaillir, pleurer, frémir, m'indigner d'abord; tu récréeras mes yeux après, si tu peux ${ }^{8}$ ». Comprendre les moyens propres à la peinture sera la tâche du critique, mais le désir du spectateur précède, d'une certaine manière, l'analyse des règles et amène plutôt à interroger l'efficacité des œuvres - littéralement leur capacité de produire des effets - et donc, pour paraphraser Michael Fried, la place consacrée au spectateur. Pour synthétiser ce premier plan d'observation adopté par Diderot, nous pouvons dire qu'il interroge le médium comme condition du regard, comme ce « qui nous permet de percevoir les images de telle sorte que nous ne les confondions ni avec de vrais corps ni avec de simples choses ». L'expression «condition du regard» pourra bien sûr sembler trop vague et on pourrait succomber à la tentation d'évoquer ici la notion de «dispositif» : ce faisant, l'on risquerait d'ajouter de la confusion plutôt que de clarifier la notion'. Nous nous limiterons à prendre en compte ici seulement deux conditions, qui ressortent souvent des pages de Diderot et qui, d'une certaine manière, peuvent nous aider à mieux comprendre la notion de médium : la manière dont le tableau s'adresse au spectateur et son contexte d'exposition. En ce sens, le médium pourrait signifier les manières par lesquelles la relation entre l'œuvre et le spectateur s'instaure.

7 "J'aime assez dans un tableau un personnage qui parle au spectateur sans sortir du sujet ${ }^{10} »$. Ce qui est étonnant et innovant dans cette remarque à propos d'un tableau de Greuze, ce n'est pas l'accent mis sur la dimension rhétorique grâce à laquelle le tableau s'adresse au spectateur, mais justement le dépassement de cette dimension rhétorique par la naturalité d'une figure qui parle au spectateur tout en restant plongée dans son activité, comme c'est le cas dans de nombreux tableaux de Chardin. Il s'agit ici de cette primauté de l'absorbement qui, selon Michael Fried, caractérise en partie la peinture française au milieu du XVIII ${ }^{\mathrm{e}}$ siècle :

L'analyse des tableaux et des textes critiques des années 1750 et du début des années 1760 révèle l'importance d'un ensemble de problèmes qui ont toujours tacitement travaillé la peinture occidentale. Je les regroupe tous sous l'expression générique de la « primauté de l'absorbement [absorption] ». Ils ont essentiellement marqué la peinture du XVII ${ }^{\mathrm{e}}$ siècle, que l'on peut rétrospectivement décrire largement comme une peinture de l'absorbement - absorbement des personnages des tableaux dans un état ou une activité. Il fallut cependant attendre le milieu des années 1750 pour voir la critique et la peinture françaises poser en toute conscience la représentation de l'absorbement visant à convaincre le spectateur comme une exigence explicite, c'est-àdire un effet spécifiquement artistique requérant une forme de plus en plus particulière de virtuosité. (L'absorbement prit forme d'exigence au moment précis où il cessa d'apparaitre comme une ressource picturale assurée) $)^{11}$.

C'est le paradoxe rhétorique que l'on retrouvera dans Le Fils naturel mais qui traverse en entier les Salons de Diderot: l'acmé de l'efficacité d'un tableau correspond à sa naturalité, à sa vérité, et cette vérité correspond à la capacité du tableau d'oublier le spectateur, c'est-à-dire d'oublier la frontière entre l'espace fictif et l'espace vécu par le spectateur. Dans son article pour l'Encyclopédie sur la composition en peinture, Diderot insistait déjà sur le but principal d'un tableau : l'unité du point de vue ${ }^{12}$. L'harmonie des rapports entre les parties n'est pas pour ainsi dire finalisée en fonction de l'équilibre du 
tableau, mais sert surtout à bâtir un angle d'observation, un seuil qui permet au spectateur de plonger instantanément dans l'espace de l'œuvre.

9 En tant que spectateur, Diderot est aussi sensible à l'accrochage des tableaux lors des Salons, d'autant plus que de 1761 à 1773, le rôle de tapissier avait été confié à son cher Chardin ${ }^{13}$. Les remarques sur l'accrochage se multiplient :

Chardin qui a été cette année ce qu'ils appellent le tapissier, à côté de ces deux misérables esquisses, en a placé une de Greuze, qui en fait cruellement la satire. C'est bien là le cas du malo vicino [...]. Excellente leçon pour le Prince dont on a entremêlé les compositions avec celles de Vernet, il ne perdra pas ce qu'il a, et il connaîtra ce qui lui manque ; [...] Ce cruel voisinage est encore une des malices du tapissier Chardin ${ }^{14}[. .$. Ce tapissier Chardin est un espiègle de la première force, il est enchanté quand il a fait quelques bonnes malices ${ }^{15}$.

Pour la première fois en histoire de l'art, l'exposition est traitée comme médium et ce n'est pas un hasard qu'un artiste de renom en soit chargé. Avec le livret du Salon comme guide, Diderot décrit avant tout à Grimm et à ses lointains correspondants couronnés son expérience de vision, y compris les effets dus à l'accrochage.

11 En spectateur, Diderot ne peut que constater les effets d'une œuvre sur lui et donc son efficacité; en critique, il doit nécessairement s'interroger sur les moyens qui permettent d'atteindre une telle efficacité. Nous pouvons identifier deux moments de cette interrogation : un premier, qui est celui du «critique militant " précisant pas à pas son lexique sans le définir; un deuxième qui est celui du "critique théorique " - celui des Essais sur la peinture ou des Pensées détachées sur la peinture, la sculpture, l'architecture et la poésie - qui vise à organiser, sans volonté de systématisation, son vocabulaire critique. En reprenant l'expression de Dieckmann, nous pourrions ajouter une troisième nuance à celles-ci, celle de critique technicien ${ }^{16}:$ celui qui s'intéresse de près aux techniques artistiques. À titre d'exemple, rappelons la publication de l'Histoire et secret de la peinture en cire, l'édition du Traité des couleurs pour la peinture en émail et sur la porcelaine de Didier-François d'Arclais de Montamy ou des Leçons de clavecin, et Principes d'harmonie d'Anton Bemetzrieder, l'attention pour les articles de l'Encyclopédie consacrés aux pratiques artistiques. En tout cas, le point crucial pour Diderot critique d'art est toujours celui présenté dans la Lettre sur les sourds et muets : comprendre les hiéroglyphes propres à chaque art. Voici donc un deuxième sens du médium que nous pouvons retrouver dans la réflexion de Diderot et que nous pouvons lire de nouveau dans une perspective moderniste : le médium comme langage propre à chaque art.

Dans la première page du Salon de 1765, celui qui contiendra aussi les Essais sur la peinture, Diderot raconte presque humblement son apprentissage critique, comment il a quitté la simple condition de spectateur, poussé par la nécessité de réfléchir sur les notions de l'art (nécessité qui le pousse à la rédaction des Essais); et il montre surtout que les termes adoptés pour décrire les tableaux correspondent au langage propre à la peinture et que chaque art possède son langage.

Si j'ai quelques notions réfléchies de la peinture et de la sculpture, c'est à vous, mon ami, que je les dois ; j'aurais suivi au Salon la foule des oisifs ; j'aurais accordé, comme eux, un coup d'œil superficiel et distrait aux productions de nos artistes ; d'un mot, j'aurais jeté dans le feu un morceau précieux, ou porté jusqu'aux nues un ouvrage médiocre, approuvant, dédaignant, sans rechercher les motifs de mon engouement ou de mon dédain. C'est la tâche que vous m'avez proposée, qui a fixé mes yeux sur la toile, et qui m'a fait tourner autour du marbre. J'ai donné le temps à l'impression d'arriver et 
d'entrer. J'ai ouvert mon âme aux effets. Je m'en suis laissé pénétrer. J'ai recueilli la sentence du vieillard et la pensée de l'enfant, le jugement de l'homme de lettres, le mot de l'homme du monde et les propos du peuple ; et s'il m'arrive de blesser l'artiste, c'est souvent avec l'arme qu'il a lui-même aiguisée. Je l'ai interrogé ; et j'ai compris ce que c'était que finesse de dessin et vérité de nature. J'ai conçu la magie de la lumière et des ombres. J'ai connu la couleur ; j'ai acquis le sentiment de la chair ; seul, j'ai médité ce que j'ai vu et entendu ; et ces termes de l'art, unité, variété, contraste, symétrie, ordonnance, composition, caractères, expression, si familiers dans ma bouche, si vagues dans mon esprit, se sont circonscrits et fixés.

ô mon ami ! que ces arts, qui ont pour objet d'imiter la nature, soit avec le discours, comme l'éloquence et la poésie ; soit avec les sons, comme la musique ; soit avec les couleurs et le pinceau, comme la peinture ; soit avec le crayon, comme le dessin ; soit avec l'ébauchoir et la terre molle, comme la sculpture ; le burin, la pierre et les métaux, comme la gravure ; le touret, comme la gravure en pierres fines; les poinçons, le matoir et l'échoppe, comme la ciselure, sont des arts longs, pénibles et difficiles ${ }^{17}$ !

Nous trouvons condensé dans ce passage, tout le parcours de Diderot qui, ayant fixé ses yeux sur la toile, commence à s'interroger sur la nature de ses sentiments et les limites de son langage, voit la continuité entre technique et théorie, et constate que sa sensibilité s'aiguise au fur et à mesure que ses idées se précisent. Mais les seules différences techniques entre les arts ne suffisent pas à comprendre l'idée d'imitation, qui met directement en jeu le rapport à la nature.

L'éclectisme et la curiosité du philosophe vont maintenant imposer à toute tentative d'analyse de sa pensée la traversée des péripéties dans les domaines les plus variés ainsi que la reconnaissance d'un fond unitaire. Bien évidemment, il ne s'agit pas de trouver une unité systématique qui a toujours été refusée par Diderot, mais plutôt une cohérence générale telle que l'on peut la chercher dans sa philosophie de la nature. Nous pourrions être tentés de dire que toute la philosophie de Diderot est une philosophie de la nature et que toute division disciplinaire a posteriori - son esthétique, sa morale - doit être intégrée au sein de sa philosophie de la nature. Il s'agit d'une nécessité de lecture souvent dictée par les textes mêmes. Comment lire autrement le très célèbre incipit des Essais sur la peinture? «La nature ne fait rien d'incorrect. Toute forme belle ou laide a sa cause, et de tous les êtres qui existent, il n'y en a pas un qui ne soit comme il doit être ${ }^{18}$ ». L'imitation de l'artiste ne doit donc pas se concentrer sur les effets de la nature mais sur les causes, pour les retrouver dans le tableau et nous faire "sentir une liaison secrète, un enchaînement nécessaire ${ }^{19}$ " entre les parties d'un corps. L'artiste doit observer les phénomènes de la nature et saisir tout ce qui produit des changements, tout ce qui introduit des variations, car il n'y a pas dans la nature de corps abstraits et idéaux, mais toujours des corps qui se transforment. La vérité d'un tableau ne dépend pas de la fidélité de la reproduction des effets, mais de sa capacité de présenter de manière analogique les relations et les causes d'une forme.

En philosophe, Diderot nous donne alors à penser un troisième sens du médium, celui du médium comme corps matériel. Il s'agit sans doute de la forme la plus complexe de médium parmi celles que nous présentons ici ; elle nécessite au moins une articulation en trois moments.

Avant tout, chaque corps possède une organisation qui lui est propre et lorsque Diderot parle de la composition en peinture il vise justement cette analogie entre le tableau et le corps vivant : « Un tableau bien composé est un tout renfermé sous un seul point de 
vue, où les parties concourent à un même but, et forment par leur correspondance mutuelle un ensemble aussi réel que celui des membres dans un corps animal ${ }^{20}$. L'être organique du tableau n'est pas simplement une règle de disposition des figures, mais le tableau comme corps vivant est la condition pour que le spectateur entre en résonance, pour réveiller "l'idée de rapports", percevoir hors de lui les rapports de son entendement.

Chaque fonction que le corps remplit exerce un effet non seulement sur une de ses parties, mais sur le corps tout entier. Il y a une « conspiration générale des mouvements ", une interdépendance de toutes les parties que l'artiste doit connaître et sentir pour les représenter [...] Ce qui est “imité" c'est la manifestation de certaines lois, l'expression de certaines fonctions ; l'une et l'autre n'existent que pour celui qui sait former l'idée d'un tout, d'un ensemble de causes et d'effets ${ }^{21}$. Le tableau imite un organisme complexe et devient ainsi un organisme vivant auquel le spectateur peut se rapporter de vivant à vivant, comme si la frontière de l'objet fictionnel pouvait être franchie grâce au sentiment de vérité, grâce au partage du sensible.

Ensuite, dès la Lettre sur les sourds et muets, Diderot montre comment tout langage, toute forme expressive doit être incorporé dans un corps : un corps qui n'est pas seulement un support mais une condition essentielle du langage. La formation du langage - et aussi la diversité des langages artistiques - dépend de nos organes de réception et d'expression. Il n'y a pas de langage sans incorporation.

Enfin, une dernière dimension ouvertement métaphysique vient outrepasser toute considération spécifique du problème artistique : le tableau en tant que corps vivant est une matière sensible et c'est justement la sensibilité qui, à partir du Rêve de D'Alembert, est considérée par Diderot comme la propriété universelle de la matière qui garantit le commercium entre les êtres, qui constitue le continuum des vivants. La réflexion de Diderot sur les arts ne peut pas être séparée de son matérialisme biologique, de ses idées sur la sensibilité de la matière et la variabilité infinie de la nature.

Ces trois positions différentes sur le médium, ces trois angles convergents d'observation, laissent en suspens un problème majeur. Qu'est-ce qui rend possible et légitime la comparaison des œuvres issues de différents arts? Le sentiment ressenti par le spectateur, les effets de chaque œuvre peuvent-ils suffire à rapprocher des œuvres et à en saisir la spécificité ?

21 Diderot écarte explicitement la possibilité que la comparaison soit fondée sur un système des beaux-arts ${ }^{22}$. Mais peut-on vraiment comparer les œuvres de différents arts sans posséder un système de référence ? En d'autres termes, peut-on véritablement parler d'intermédialité sans avoir recours à un système implicite ou explicite? Sans un principe unitaire, une source commune aux divers hiéroglyphes? La critique que Diderot fait du système de Batteux ne cache-t-elle pas une exigence plus profonde que ses remarques sur le caractère vague de la belle nature et de la bonne imitation?

\section{Les hiéroglyphes vs le système des beaux-arts}

De la Lettre sur les sourds et muets, nous l'avons vu auparavant, Lessing avait apprécié surtout la richesse d'idées de Diderot, malgré l'absence de rigueur dans l'argumentation, trop légèrement justifiée par le choix de la forme épistolaire. 
Cependant, le passage désinvolte d'un argument à un autre révèle peut-être un aspect structurel de la pensée de Diderot. Comme le soulignait Paul Hugo Meyer qui, en 1965, avait dirigé une importante édition critique du texte, «le manque de composition rigoureuse de la Lettre témoigne simplement du fait que pour Diderot il n'y a pas au fond de distinction organique acceptable entre métaphysique et esthétique, ou encore entre pensée et sensibilitée ${ }^{3}$ " et, si Diderot "vire en cours de chemin de la métaphysique à l'esthétique, c'est parce que ce passage correspond à celui qui conduit les idées de leur naissance à leur expression ${ }^{24}$ ». C'est justement dans cette profonde unité de la métaphysique et de l'esthétique qu'il faut intégrer la réflexion de Diderot sur la spécificité et la singularité des médiums artistiques.

Parfois, souvent même, on cite la Lettre pour y retrouver la critique assez féroce que Diderot adresse à Batteux, destinataire de la lettre, en particulier à son traité Les BeauxArts réduits à un même principe qui, dès sa parution en 1746, avait connu un succès extraordinaire. Contre le système des beaux-arts de Batteux, Diderot défend, dans la ligne de Shaftesbury et de Dubos, les différences entre poésie et peinture, et, plus généralement, les « hiéroglyphes particuliers » de tout art d'imitation, qui conduisent plutôt à un travail de comparaison qu'à une tentative de réduction à un seul principe. Lisons ce passage célèbre de la Lettre:

Partout où l'hiéroglyphe accidentel aura lieu, soit dans un vers, soit sur un obélisque, comme il est ici l'ouvrage de l'imagination, et là celui du mystère, il exigera pour être entendu, ou une imagination ou une sagacité peu communes. Mais s'il est si difficile de bien entendre des vers, combien ne l'est-il pas davantage d'en faire ? On me dira peutêtre tout le monde fait des vers; et je répondrai simplement, presque personne ne fait des vers. Tout art d'imitation ayant ses hiéroglyphes particuliers, je voudrais bien que quelque esprit instruit et délicat s'occupât un jour à les comparer entre eux. Balancer les beautés d'un poète avec celles d'un autre poète, c'est ce qu'on a fait mille fois. Mais rassembler les beautés communes de la poésie, de la peinture et de la musique, en montrer les analogies, expliquer comment le poète, le peintre et le musicien rendent la même image, saisir les emblèmes fugitifs de leur expression, examiner s'il n'y aurait pas quelque similitude entre ces emblèmes, etc. c'est ce qui reste à faire, et ce que je vous conseille d'ajouter à vos Beaux-Arts réduits à un même principe. Ne manquez pas non plus de mettre à la tête de cet ouvrage un chapitre sur ce que c'est la belle nature; car je trouve des gens qui me soutiennent que faute de l'une de ces choses votre traité reste sans fondement ; et que faute de l'autre, il manque d'application. Apprenez-leur, Monsieur, une bonne fois comment chaque art imite la nature dans un même objet; et démontrez-leur qu'il est faux, ainsi qu'ils le prétendent, que toute nature soit belle, et qu'il n'y ait de laide nature que celle qui n'est pas à sa place. ${ }^{25}$

Pour illustrer sa position, Diderot se lance ensuite dans la comparaison de trois œuvres - quelques vers de l'Enéide, une gravure de Frans Van Mieris et un fragment de musique composé par Diderot même - qui ont le même objet d'imitation, une femme mourante. Voici donc ce que Diderot propose à la place d'un système abstrait sans fondement ni application : un travail de terrain en contact direct avec les œuvres dans leur singularité, prenant en compte leur processus de fabrication. Même si le fond la Lettre reste métaphysique, nous pouvons déjà y trouver les présupposés qui seront mis en œuvre dans critique des Salons ${ }^{26}$. Et il lance ainsi une nouvelle pique à l'abbé Batteux : 
Je ne doute point que l'on ne trouvât dans nos peintres, nos poètes et nos musiciens des exemples, et plus analogues encore les uns aux autres et plus frappants du sujet même que j'ai choisi : mais je vous laisse le soin de les chercher et d'en faire usage, à vous, Monsieur, qui devez être peintre, poète, philosophe et musicien ; car vous n'auriez pas tenté de réduire les beaux-arts à un même principe, s'ils ne vous étaient pas tous à peu près également connus ${ }^{27}$.

Ne serait-ce que pour la verve de Diderot face au style plutôt austère de Batteux, on peut facilement être tenté de sympathiser avec le Philosophe dans cette querelle, en courant le risque de simplifier la réflexion de Diderot ainsi que l'apport de Batteux, et de tomber dans quelques contradictions.

Tout d'abord, Diderot ne nie pas la notion de beaux-arts ni leur unité générale donnée par l'imagination. En d'autres termes, il n'a jamais remis en question le système des connaissances du Discours préliminaire et la place des beaux-arts dans ce système :

La peinture, la sculpture, l'architecture, la poésie, la musique et leurs différentes divisions, composent la troisième distribution générale, qui naît de l'imagination, et dont les parties sont comprises sous le nom de beaux-arts. On pourrait aussi les renfermer sous le titre général de peinture, puisque tous les beaux-arts se réduisent à peindre, et ne diffèrent que par les moyens qu'ils emploient; enfin, on pourrait les rapporter tous à la poésie, en prenant ce mot dans sa signification naturelle, qui n'est autre chose qu'invention ou création ${ }^{28}$.

Pareillement, la notion d'arts d'imitation n'a jamais été remise en cause. Ce que Diderot critique chez Batteux est une idée abstraite d'imitation qui ne rend pas compte des différences des formes artistiques et de la singularité des observations des artistes sur la nature. Et c'est justement la question de la «belle nature» qui pose le plus de problèmes à Diderot et qui nous montre la profonde continuité entre métaphysique et esthétique dans sa pensée. Toute nature est belle et il n'y a pas de nature figée. «Tout ce qui est ne peut être ni contre nature ni hors de nature ", dira Bordeau dans le Rêve de D'Alembert. La nature ne donne pas à voir des objets finis mais des processus, des rapports de cause à effet, des relations. L'artiste doit observer les transformations toujours en acte des formes naturelles, en saisir les causes et retrouver avec le langage propre à son art le système de relations visé.

Pour approfondir l'étude des critiques formulées contre Batteux, il ne faut pas oublier qu'elles apparaissent dans la dernière partie de l'ouvrage, après l'analyse de la question de l'inversion linguistique (qui était, l'argument qui précède le texte le déclare ${ }^{29}$, l'objet principal de la Lettre) et l'introduction de l'idée de hiéroglyphe.

Qu'est-ce que le hiéroglyphe ? Il est le stade le plus avancé de perfectionnement d'un langage et c'est à ce stade que Diderot reconnaît l'émergence et la spécificité du phénomène poétique ${ }^{30}$. Pour arriver à ce stade il faut suivre une évolution que Diderot décrit dans ces termes: "Il faut distinguer dans toutes les langues trois états par lesquels elles ont passé successivement au sortir de celui où elles n'étaient qu'un mélange confus de cris et de gestes, mélange qu'on pourrait appeler du nom de langage animal. Ces trois états sont l'état de naissance, celui de formation, et l'état de perfection ${ }^{31}$ ». Cette ligne évolutive sert à démontrer la distinction nette qui subsiste pour Diderot entre l'ordre naturel des idées et l'ordre d'institution du langage : « Je dis l'ordre naturel des idées ; car il faut distinguer ici l'ordre naturel d'avec l'ordre d'institution, et pour ainsi dire, l'ordre scientifique; celui des vues de l'esprit, lorsque la langue fut tout à fait formée $^{32} »$. C'est certainement parmi les propositions les plus originales de la Lettre : la 
formation des idées est indépendante de l'évolution du langage et le degré de perfectionnement de ce dernier n'est pas déterminé par sa correspondance avec l'ordre des idées mais par sa capacité analytique et, pour ainsi dire, générative et expressive. Pensée et langage ne naissent pas ensemble et ne se développent pas simultanément et le sourd-muet en est la preuve : « un homme qui s'interdisant l'usage des sons articulés, tâcherait de s'exprimer par gestes ${ }^{33}$ ".

31 L'évolution d'une langue, selon Diderot, pourrait se résumer ainsi : sur la base d'un «langage animal» qui peut compter seulement sur les signes naturels de cris et de gestes, se fonde le stade de naissance du langage humain qui utilise encore les gestes et des mots invariables, et qui suit l'ordre naturel des idées. Le deuxième état, celui de la formation, est caractérisé par l'abandon des gestes et la découverte d'une harmonie syllabique. Dans le troisième stade, celui de la perfection « on a voulu de plus de l'harmonie, parce qu'on a cru qu'il ne serait pas inutile de flatter l'oreille en parlant à l'esprit » et « comme on préfère souvent l'accessoire au principal ; souvent aussi l'on a renversé l'ordre des idées pour ne pas nuire à l'harmonie ${ }^{34}$ ».

Le hiéroglyphe représente le moment strictement poétique où l'accessoire ${ }^{35}$ est devenu l'élément principal et génère une nouvelle production de sens qui dépasse la signification des mots. L'expérience qu'on peut faire du hiéroglyphe est une expérience esthétique totale, dans laquelle nos sens, nos sentiments, notre imagination et notre entendement sont pareillement sollicités :

Il passe alors dans le discours du poète un esprit qui en meut et vivifie toutes les syllabes. Qu'est-ce que cet esprit ? J'en ai quelquefois senti la présence ; mais tout ce que j'en sais, c'est que c'est lui qui fait que les choses sont dites et représentées tout à la fois; que dans le même temps que l'entendement les saisit, l'âme en est émue, l'imagination les voit, et l'oreille les entend ; et que le discours n'est plus seulement un enchaînement de termes énergétiques qui exposent la pensée avec force et noblesse, mais que c'est encore un tissu d'hiéroglyphes entassés les uns sur les autres qui la peignent. Je pourrais dire en ce sens que toute poésie est emblématique. Mais l'intelligence de l'emblème poétique n'est pas donnée à tout le monde ; il faut être presque en état de le créer pour le sentir fortement ${ }^{36}$.

Ainsi le spectateur qui parvient à saisir avec toutes ses facultés l'emblème devient luimême l'artiste. D'une certaine manière, on serait tenté de dire que le hiéroglyphe conduit circulairement à un état pré-linguistique, mais cette lecture pousserait peutêtre un peu trop loin les intentions de Diderot. Ce qui est certain c'est que le hiéroglyphe représente le moment où, après avoir distingué et analysé les idées, et après avoir structuré un langage, nous pouvons retrouver la profonde unité entre sensation, sentiment, imagination et entendement.

"Chaque art a ses avantages; il semble qu'il en soit d'eux comme des sens. Les sens ne sont tous qu'un toucher; tous les arts, qu'une imitation. Mais chaque sens touche, et chaque art imite d'une manière qui lui est propre $^{37} »$. Toute forme expressive peut atteindre un état de perfection qui produit du sens et conduit à l'unité nos processus d'appréhension du monde. C'est la découverte de ce qui est strictement artistique qui transforme un problème artistique en une question métaphysique. En ce sens, en considérant le hiéroglyphe comme le médium propre à un art, nous pouvons lire ce passage assez étonnant de la Lettre:

Je fréquentais jadis beaucoup les spectacles, et je savais par cœur la plupart de nos bonnes pièces. Les jours que je me proposais un examen des mouvements et du geste, 
j'allais aux troisièmes loges ; car plus j'étais éloigné des acteurs, mieux j'étais placé. Aussitôt que la toile était levée, et le moment venu où tous les autres spectateurs se disposaient à écouter ; moi, je mettais mes doigts dans mes oreilles, non sans quelque étonnement de la part de ceux qui m'environnaient, et qui ne me comprenant pas, me regardaient presque comme un insensé qui ne venait à la comédie que pour ne la pas entendre. Je m'embarrassais fort peu des jugements, et je me tenais opiniâtrement les oreilles bouchées, tant que l'action et le jeu de l'acteur me paraissaient d'accord avec le discours que je me rappelais. Je n'écoutais que quand j'étais dérouté par les gestes, ou que je croyais l'être. [...] Quoi que vous pensiez de mon expédient, je vous prie de considérer que, si pour juger sainement de l'intonation, il faut écouter le discours sans voir l'acteur ; il est tout naturel de croire que pour juger sainement du geste et des mouvements, il faut considérer l'acteur sans entendre le discours ${ }^{38}$.

\section{Promenade au Salon}

Diderot a été un homme de théâtre et le paradigme de la scène a toujours été prépondérant dans sa réflexion sur les $\operatorname{arts}^{39}$. Et au théâtre, il ne se contente pas de se boucher les oreilles, il arrive même à imaginer le "quatrième mur » qui laisse aux acteurs la possibilité d'oublier complètement la présence du public : «Imaginez sur le bord du théâtre un grand mur qui vous sépare du parterre; jouez comme si la toile ne se levait pas $^{40}$ ». Encore un changement de perspective, un bouleversement du point de vue, une expérience mentale visant à dévoiler les traits fondamentaux d'un phénomène artistique. Eisenstein, presque deux siècles plus tard, montrera comment selon lui « seul le cinéma réalise jusqu'au bout [ce] précepte de Diderot ${ }^{41}$ ».

En sourd, il peut aller au théâtre, en sourd - en reprenant un topos des théories de la peinture - il peut même envisager de visiter une exposition, pour saisir les gestes, les mouvements des figures: "Celui qui se promène dans une galerie de peintures fait, sans y penser, le rôle d'un sourd qui s'amuserait à examiner des muets qui s'entretiennent sur des sujets qui lui sont connus ${ }^{42} »$.

Diderot tout aussi curieux du bavardage du public des Salons, rapporte parfois les petits commentaires volés dans la foule, aime à observer les effets que les œuvres exercent sur les gens. Mais il s'est surtout " laissé pénétrer " par les œuvres et leurs effets. Avant d'être critique et philosophe, il a été spectateur et en tant que spectateur qui ouvre son âme, il s'interroge parfois sur la médiation, sur l'échange entre l'espace de la peinture et son espace vécu. C'est en ce sens que nous pouvons reconnaître chez Diderot une idée de médium comme condition du regard qui concerne la prédisposition du spectateur, le sujet du tableau, la disposition de l'exposition.

La promenade n'est plus alors une simple déambulation, mais la synthèse même de l'activité du spectateur: on se promène dans la nature comme dans un tableau, on se promène dans le tableau comme à la campagne, on se promène au Louvre avec le livret du Salon en main et on observe les effets de montage de l'exposition. C'est surtout l'expérience de celui qui est devenu spectateur en étant passé par le stade du critique et du philosophe pour affiner sa sensibilité.

Une des plus belles et célèbres pages des Essais sur la peinture résume le sens de ce type de promenade :

S'il nous arrive de nous promener aux Tuileries, au bois de Boulogne, ou dans quelque endroit écarté des Champs Elysées sous quelques-uns de ces vieux arbres épargnés 
parmi tant d'autres qu'on a sacrifiés au parterre et à la vue de l'hôtel de Pompadour, sur la fin d'un beau jour, au moment où le soleil plonge ses rayons obliques à travers la masse touffue de ces arbres dont les branches entremêlées les arrêtent, les renvoient, les brisent, les rompent, les dispersent sur les troncs, sur la terre, entre les feuilles, et produisent autour de nous une variété infinie d'ombres fortes, d'ombres moins fortes, de parties obscures, moins obscures, éclairées, plus éclairées, tout à fait éclatantes ; alors les passages de l'obscurité à l'ombre, de l'ombre à la lumière, de la lumière au grand éclat, sont si doux, si touchants, si merveilleux, que l'aspect d'une branche, d'une feuille arrête l'œil, et suspend la conversation au moment même le plus intéressant. Nos pas s'arrêtent involontairement ; nos regards se promènent sur la toile magique, et nous nous écrions : quel tableau! Oh que cela est beau ! Il semble que nous considérions la nature comme le résultat de l'art. Et réciproquement, s'il arrive que le peintre nous répète le même enchantement sur la toile, il semble que nous regardions l'effet de l'art comme celui de la nature. Ce n'est pas au Salon, c'est dans le fond d'une forêt, parmi les montagnes que le soleil ombre et éclaire, que Loutherbourg et Vernet sont grands ${ }^{43}$.

Starobinski observe à juste titre le paradoxe : « Pour trouver la vérité, il faudrait quitter Paris, vivre à la campagne. Mais il faut habiter les villes pour acquérir les qualités essentielles de l'artiste ${ }^{44} »$. C'est le paradoxe de la vérité en peinture ou autrement dit le rêve de Diderot de surmonter le conflit entre nature et culture. C'est le même paradoxe que celui de la « promenade Vernet » du Salon de 1767.

41 La promenade devient aussi, toujours en 1767, une sorte de méthode descriptive ou au moins une rhétorique du critique qui cherche une correspondance entre le mouvement du spectateur et le discours du tableau : "C'est une assez bonne méthode pour décrire des tableaux, surtout champêtres, que d'entrer sur le lieu de la scène par le côté droit ou par le côté gauche, et s'avançant sur la bordure d'en bas, de décrire les objets à mesure qu'ils se présentent. Je suis bien fâché de ne m'en être pas avisé plus tôt ${ }^{45}$ ».

En conclusion, revenons un instant sur une phrase de la précédente citation des Essais : "...l'aspect d'une branche, d'une feuille arrête l'œil, et suspend la conversation... ». « Pour Diderot - écrit encore Starobinski - la peinture digne d'être aimée est celle qui l'“arrête" et le "fixe", celle qui l'oblige à revenir sans cesse à une scène éloquente ou magique, et c'est tout ensemble celle qui l'incite à inventer, en écho, des images qui n'ont pas encore trouvé leur peintre, et que le langage s'ingénie à tracer avec ses ressources propres, à la fois surabondantes et pauvres ${ }^{46} »$.

L'instantanéité du point de vue, l'arrêt sur le tableau, la suspension du discours, nous positionnent à la place du peintre $e^{47}$, nous conduisent au point de départ de notre promenade dans le monde, au moment qui précède le long travail du pinceau et l'analyse des idées conduite par le langage : le moment où sentir et juger coïncident, où nous adhérons pleinement au monde de la vie. 


\section{BIBLIOGRAPHIE}

Alembert Jean Le Rond d', Discours Préliminaire, éd. Michel Malherbe, Paris, Vrin, 2000.

Altachina Véronika, « Eisenstein, lecteur de Diderot », Recherches sur Diderot et sur l'Encyclopédie, 50, 2015., p. 155-165

Arbo Alessandro, « Diderot et l'hiéroglyphe musical », Recherches sur Diderot et sur l'Encyclopédie, 30,2001 , p. 65-80.

Barthes Roland, « Diderot, Brecht, Eisenstein », Revue d'esthétique, 2-3-4, 1973, repris dans L'Obvie et l'obtus, Essais Critiques III, Paris, éditions du Seuil, 1982, p. 148-160.

Batteux Charles, Les Beaux-Arts réduits à un même principe, (1746) éd. critique par Jean-Rémy Mantion, Paris, Aux amateurs de livres, 1989.

Becq Annie, « Diderot Historien de l'art ? ", Dix-huitième siècle, 19, 1987, p. 423-438.

Belleguic Thierry, «La matière de l'art Diderot et l'expérience esthétique dans les premiers "Salons" ", Diderot Studies, 30, 2007, p. 3-10.

Belting Hans, Bild-Anthropologie : Entwürfe für eine Bildwissenschaft, Munich, Fink, 2001 ; trad. par Jean Torrent, Pour une anthropologie des images, Paris, Gallimard, 2004.

Bonnet Jean-Claude, « Diderot a inventé le cinéma », Recherches sur Diderot et sur l'Encyclopédie, numéro 18-19, 1995, p. 27-33.

Boulerie Florence, « Diderot et le vocabulaire technique de l'art : Des premiers "Salons" aux "Essais sur la peinture" ", Diderot Studies, 30, 2007, p. 89-111.

Buchs Arnaud, «Quand le tableau se fait image. Diderot en ses Salons », Poétique, 160, 2009, p. 405-415.

Cartwright Michael T., « Diderot critique d'art et le problème de l'expression », Diderot Studies, 13, 1969, p. V-XII, 1-267.

Chouillet Jacques, La formation des idées esthétiques de Diderot, Paris, Armand Colin, 1973.

Cohen Huguette, «Diderot et les limites de la littérature dans les Salons », Diderot Studies, 24, 1991, p. 25-45.

Delon Michel, « Le regard détourné Diderot et les limites de la représentation », dans Diderot, Denis, Écrits sur l'art et les artistes, textes réunis et présentés par Jean Seznec, Paris, Hermann, 2007, p. 259-275.

Delon Michel, « Les "Essais sur la peinture" ou la place de la théorie », Diderot Studies, 30-2007, p. 31-51.

Di Liberti Giuseppe, "Diderot : Le système des arts comme système des sens ", dans MariePauline Martin et Chiara Savettieri (dir.), La musique face au système des beaux arts ou les vicissitudes de l'imitation (1690-1803), Paris, Vrin, 2014, p. 57-65.

Di Liberti Giuseppe, Il sistema delle arti. Storia e ipotesi, Milan, Mimesis, 2009 ; trad. par Agathe Henssien et Pauline Rougier, Le système des arts, traduction revue et augmentée, Paris, Vrin, 2016.

Diderot Denis, CEuvres, édition établie par Laurent Versini en 5 tomes, Paris, Robert Laffont, 1994-1997, tome IV : Esthétique - Théâtre, 1996. Abréviation adoptée en note : LV, 4.

Dieckmann Herbert, Cinq leçons sur Diderot, Genève, Droz, 1959. 
Duflo Colas, Diderot philosophe, Paris, Honoré Champion, 2003.

Eisenstein Sergueï, « Diderot a parlé de cinéma », Europe, 1984, 661, p. 133-141.

Fried Michael, Absorption and Theatricality Painting and Beholder in the Age of Diderot, Berkeley, University of California Press, 1980 ; trad. par Claire Brunet, La place du spectateur, Paris, Gallimard, 1990.

Funt David, « Diderot and the Esthetics of the Enlightenment », Diderot Studies, 11, 1968, p. 5-192. Genand Stéphanie, «L'œil ravi : violences du regard dans les premiers "Salons" de Diderot », Diderot Studies, 30, 2007, p. 143-154.

Hobson Marian, «Quelques Références dans la "Lettre sur les sourds et muets” ", Diderot Studies, 18, 1975, p. 111-119.

Lavezzi Élisabeth, « Remarques sur la peinture dans la Lettre sur les sourds et muets », Recherches sur Diderot et sur l'Encyclopédie, 46, 2011, p. 72-84.

Lessing Gotthold Ephraim, Laocoon, trad. française de Courtin [1866], revue et corrigée, Paris, Hermann, 2002.

Lojkine Stéphane, «Le problème de la description dans les "Salons" de Diderot », Diderot Studies, 30, 2007, p. 53-72.

Lojkine Stéphane, «Vérité, poésie, magie de l’art : les Salons de Diderot », cours donné à l'université de Provence, sept.-déc. 2011, en ligne http://utpictura18.univ-montp3.fr/

May Georges, « À l'usage de ceux qui lisent la Lettre sur les sourds et muets », Diderot Studies, 7, " Diderot: "Lettre sur les sourds et muets" ", Edition commentée et présentée par Paul Hugo Meyer, 1965, p. V-XXVI.

May Gita, Diderot et Baudelaire critiques d'art, Genève, Droz, 1973.

Meyer Paul H., « Introduction », Diderot Studies, « Diderot : "Lettre sur les sourds et muets" ", Edition commentée et présentée par Paul Hugo Meyer, 7, 1965, p. 3-33.

Meyer Paul H., « The "Lettre sur les sourds et muets" and Diderot's Emerging Concept of the Critic », Diderot Studies, 6, 1964, p. 133-155.

Modica Massimo, « Diderot philosophe et critique d'art. Essai sur l'esthétique de Diderot », Recherches sur Diderot et sur l'Encyclopédie, 33, 2002, p. 73-95.

Mortier Roland, Diderot en Allemagne (1750-1850), Genève, Paris, Slaktine Reprints, 1986.

Quintili Paolo, « La couleur, la téchne, la vie. L'esthétique épistémologique des Salons

(1759-1781) », Recherches sur Diderot et sur l'Encyclopédie, 25, 1998, p. 21-39.

Starobinski Jean, «L'espace des peintres », dans Diderot dans l'espace des peintres, Paris, Réunion des musées nationaux, 1991, p. 9-68; puis dans Diderot, un diable de ramage, Paris, Gallimard, 2012, p. 335-375.

Vouilloux Bernard, « La critique des dispositifs », Critique, 718, 2007, p. 152-168.

\section{NOTES}

1. Gotthold Ephraim Lessing, Laocoon, trad.fr. par Courtin [1866], revue et corrigée, Paris, Hermann, 2002, p. 41. Derrière la figure de l'amateur se cachait très probablement l'ami Christoph Friedrich Nicolai, proche du cercle berlinois de Lessing, éditeur de la revue Bibliothek 
der schönen Wissenschaft und freyn Künste. Le philosophe était très certainement Moses Mendelssohn, qui avait publié en 1757 dans la revue de Nicolai ses Betrachtungen über die Quellen und Verbindungen der schönen Künste und Wissenschaften (remaniées en 1761 sous le titre Über die Hauptgrundsätze der schönen Künste und Wissenschaft). Et bien évidemment, le critique d'art n'est autre que Lessing lui-même, qui prenait ainsi en charge la fonction de l'analyse ponctuelle et factuelle des œuvres comme moyen pour comprendre les caractères propres de la poésie et la peinture.

2. Cité dans Roland Mortier, Diderot en Allemagne (1750-1850), Genève/Paris, Slaktine Reprints, 1986, p. 345.

3. Paul Hugo Meyer, "Introduction», Diderot Studies, "Diderot: "Lettre sur les sourds et muets" ", édition commentée et présentée par Paul Hugo Meyer, 7, 1965, p. 24.

4. Denis Diderot, Salon de 1763, CEuvres, édition établie par Laurent Versini en 5 tomes, Paris, Robert Laffont, 1994-1997, tome IV : Esthétique - Théâtre, 1996, p. 240. Abréviation adoptée en note : LV, 4 .

5. Herbert Dieckmann, Cinq leçons sur Diderot, Genève, Droz, 1959, p. 148.

6. On pourrait lire en ce sens son enthousiasme pour Chardin ou sa distance vis-à-vis de Boucher: «Quelles couleurs! quelle variété ! quelle richesse d'objets et d'idées! Cet homme a tout, excepté la vérité » (D. Diderot, Salon de 1761, LV, 4, op. cit., p. 205).

7. D. Diderot, Salon de 1763, LV, 4, op. cit., p. 289.

8. D. Diderot, Essais sur la peinture, LV, 4, op. cit., p. 498.

9. Cf. Bernard Vouilloux, « La critique des dispositifs ", Critique, 718, 2007.

10. D. Diderot, Salon de 1761, LV, 4, op. cit., p. 228.

11. Michael Fried, La place du spectateur. Esthétique et origines de la peinture moderne [1980], Paris, Gallimard, traduit de l'anglais (États-Unis) par Claire Brunet, 1990, p. 18.

12. Cf. D. Diderot, "Composition en peinture ", LV, 4, op. cit., p. 120 et aussi la note 22 . Fried souligne comment «Diderot ajoute une quatrième unité aux traditionnelles unités d'action, de temps et de lieu: celle du point de vue. Il l'introduit dès le début dans sa définition de la composition picturale et la définit avec force dans les Essais " (M. Fried, La place du spectateur, op. cit., p. 95).

13. Sur la fonction du tapissier voir Stéphane Lojkine, « Vérité, poésie, magie de l'art : les Salons de Diderot»[cours], université de Provence, sept.-déc.2011, en ligne http://utpictura18.univmontp3.fr/.

14. D. Diderot, Salon de 1765, LV, 4, op. cit., p. 320 et p. 357.

15. D. Diderot, Salon de 1769, LV, 4, op. cit., p. 869.

16. D. Diderot, Pensées détachées sur la peinture, LV, 4, op. cit., p. 1044 : « quoique toute ma réflexion soit tournée vers les principes spéculatifs de l'art, cependant, lorsque je rencontre quelques procédés qui tiennent à sa magie pratique, je ne puis m'empêcher d'en faire note ».

17. D. Diderot, Salon de 1765, LV, 4, p. 291.

18. D. Diderot, Essais sur la peinture, LV, 4, p. 467.

19. Ibid., p. 468.

20. D. Diderot, « Composition en peinture », LV, 4, p. 120.

21. H. Dieckmann, Cinq leçons sur Diderot, p. 118 ; cit. in Fried, La place du spectateur, op. cit., p. 88 qui continue: «comme Diderot y insiste, l'ensemble du tableau ne doit être qu'un système expressif et dramatique de causes et d'effets ; [...] En un mot, si l'unité picturale était une espèce de microcosme du système causal de la nature et de l'univers, l'unité de la nature appréhendée par l'homme était réciproquement, à l'instar de celle des tableaux, fondamentalement dramatique et expressive ».

22. Je me permets ici de renvoyer à mon article «Diderot: Le système des arts comme système des sens ", dans Marie-Pauline Martin et Chiara Savettieri (dir.), La musique face au système des arts 
ou les vicissitudes de l'imitation au siècle des Lumières (1690-1803), Paris, Vrin, 2014 et à mon livre Le système des arts, Paris, Vrin, 2016.

23. Gita May, « À l'usage de ceux qui lisent la Lettre sur les sourds et muets », Diderot Studies, 7, 1965, p. XXII.

24. Ibid., p. XX.

25. D. Diderot, Lettre sur les sourds et muets, LV, 4, op. cit., p. 43 ; Diderot présente la même critique à Batteux dans l'article Beau de 1752 publié ensuite en 1772 sous le titre Traité du beau: «M. l'abbé Batteux rappelle tous les principes des beaux Arts à l'imitation de la belle nature; mais il ne nous apprend point ce que c'est que la belle nature » (LV, 4, op. cit., p. 91)

26. Cf. P. H. Meyer, "The "Lettre sur les sourds et muets" and Diderot's Emerging Concept of the Critic », Diderot Studies, 6, 1964, p. 133-155.

27. D. Diderot, Lettre sur les sourds et muets, LV, 4, op. cit., p. 47.

28. Jean Le Rond D’Alembert, Discours Préliminaire, Paris, Vrin, 2000, p. 114.

29. "Où l'on traite de l'origine des inversions; de l'harmonie du style; du sublime de situation; de quelques avantages de la langue française sur la plupart des langues anciennes et modernes, et, par occasion, de l'expression particulière aux beaux-arts » (D. Diderot, Lettre sur les sourds et muets, LV, 4, op. cit., p. 13).

30. Une analyse très claire du modèle d'évolution du langage de la Lettre est offerte par Élizabeth Lavezzi, « Remarques sur la peinture dans la Lettre sur les sourds et muets », Recherches sur Diderot et sur l'Encyclopédie, 46, 2011, p. 72-84.

31. D. Diderot, Lettre sur les sourds et muets, LV, 4, op. cit., p. 32.

32. Ibid., p. 14.

33. Ibid., p. 14.

34. Ibid., p. 33.

35. E. Lavezzi, « Remarques sur la peinture dans la Lettre sur les sourds et muets», op. cit., p. 82 : «Ce que, dans les Essais, Diderot appelle accessoire, (ce qui n'est pas du tout le sens du terme en peinture) correspond au hiéroglyphe pictural de la Lettre : la représentation d'un objet visible qui intensifie l'expression et l'harmonie; le passage des Essais précise que l'énergie est apportée à l'œuvre par l'analogie entre l'objet représenté (l'arbre) et le sujet du tableau, la chaumière et la pauvreté de l'habitant qui l'occupe. Autrement dit, le vieil arbre près de la chaumière relève de la métaphore (il est misérable comme l'habitant) et de la métonymie (il est voisin de l'habitant de la chaumière). [...] si l'accessoire est un hiéroglyphe pictural, ce dernier est la représentation d'un objet choisi pour les idées qui lui sont attachées et qui s'ajoutent au sujet traité pour le renforcer. De cette façon, Diderot valorise ce qui pourrait passer pour secondaire dans un tableau (le vieil arbre n'est pas le sujet), justifiant le choix paradoxal du terme accessoire ».

36. D. Diderot, Lettre sur les sourds et muets, LV, 4, op. cit., p. 34.

37. D. Diderot, Entretiens sur le fils naturel, LV, 4, op. cit., p. 1186.

38. D. Diderot, Lettre sur les sourds et muets, LV, 4, op. cit., p. 21.

39. Roland Barthes, «Diderot, Brecht, Eisenstein », dans L'Obvie et l'obtus, Essais Critiques III, Paris, éditions du Seuil, 1982, p. 150: «Toute l'esthétique de Diderot, on le sait, repose sur l'identification de la scène théâtrale et du tableau pictural : la pièce parfaite est une succession de tableaux, c'est-à-dire une galerie, un salon: la scène offre au spectateur «autant de tableaux réels qu'il y a dans l'action de moments favorables au peintre ». Le tableau (pictural, théâtral, littéraire) est un découpage pur, aux bords nets, irréversible, incorruptible, qui refoule dans le néant tout son entour, innommé, et promeut à l'essence, à la lumière, à la vue, tout ce qu'il fait entrer dans son champ ; cette discrimination démiurgique implique une haute pensée : le tableau est intellectuel, il veut dire quelque chose (de moral, de social), mais aussi il dit qu'il sait comment il faut le dire; il est à la fois significatif et propédeutique, impressif et réflexif, émouvant et conscient des voies de l'émotion. ».

40. D. Diderot, De la poésie dramatique, LV, 4, p. 1310. 
41. Sergueï Eisenstein, «Diderot a parlé de cinéma », Europe, 1984, 661, p. 138. Sur Eisenstein lecteur de Diderot, voir Jean-Claude Bonnet, «Diderot a inventé le cinéma ", Recherches sur Diderot et sur l'Encyclopédie, numéro 18-19, 1995, p. 27-33 et Véronika Altachina, « Eisenstein, lecteur de Diderot », Recherches sur Diderot et sur l'Encyclopédie, 50, 2015, p. 155-165.

42. D. Diderot, Lettre sur les sourds et muets, LV, 4, p. 20.

43. D. Diderot, Essais sur la peinture, LV, 4, p. 478.

44. Jean Starobinski, «L'espace des peintres », dans Diderot, un diable de ramage, Paris, Gallimard, 2012, p. 340.

45. D. Diderot, Salon de 1767, LV, 4, p. 677.

46. J. Starobinski, « L'espace des peintres », op. cit., p. 373.

47. D. Diderot, Lettre sur les sourds et muets, LV, 4, p. 30 : " notre âme est un tableau mouvant d'après lequel nous peignons sans cesse : nous employons bien du temps à le rendre avec fidélité ; mais il existe en entier et tout à la fois : l'esprit ne va pas à pas comptés comme l'expression. Le pinceau n'exécute qu'à la longue ce que l'œil du peintre embrasse tout d'un coup. La formation des langues exigeait la décomposition; mais voir un objet, le juger beau, éprouver une sensation agréable, désirer la possession, c'est l'état de l'âme dans un même instant ».

\section{RÉSUMÉS}

Chez Diderot, il n'y a pas une théorie explicite du médium et bien évidemment il n'emploie jamais ce terme. Cependant, Diderot a été spectateur, critique et philosophe à la fois. À ces trois figures théoriques correspondent trois pistes d'interrogation sur la notion de médium: le médium comme condition du regard, comme langage propre à chaque art, comme corps matériel de l'œuvre. Ces trois dimensions émergent surtout dans les tentatives de Diderot de définir la place du spectateur, de reconnaitre la spécificité de chaque art et de décrire les œuvres dans leur singularité, en jetant ainsi les fondements d'une analyse comparative qui s'oppose à la fermeture du système des beaux-arts de Batteux. À partir d'une lecture de la Lettre sur les sourds et muets (et en particulier de la réflexion autour de la notion de hiéroglyphe) et de quelques passages des Salons, l'article vise à montrer comment se manifestent dès ce moment fondateur de la critique d'art la complexité et l'hétérogénéité de la notion de médium qui échappe aujourd'hui encore à toute définition unitaire.

\section{INDEX}

Mots-clés : Diderot, médium, Salons, intermédialité, hiéroglyphes, peinture, esthétique, système des beaux-arts, nature

\section{AUTEUR}

\section{GIUSEPPE DI LIBERTI}

Giuseppe Di Liberti est maître de conférences en esthétique à l'université d'Aix-Marseille et membre du CEPERC (UMR 7304). Ses travaux portent notamment sur l'histoire des idées 
esthétiques, sur les relations entre esthétique et sciences de la vie, et sur le statut de l'objet artistique. Il a publié Il sistema delle arti. Storia e ipotesi (Milan, Mimesis, 2009 ; trad. fr. Le système des arts. Histoire et hypothèse, Paris, Vrin, 2016) ; l'édition italienne du Cabinet des Beaux-arts de Charles Perrault (Palerme, Centro Internazionale Studi di Estetica, 2009) et, avec Danièle Cohn, l'anthologie Textes clés d'esthétique. Connaissance, Art, Expérience (Paris, Vrin, 2012). Il est l'auteur d'une vingtaine d'articles parus en Italie et en France. Il est parmi les membres fondateurs de la revue en ligne Images Re-vues dont il a dirigé deux numéros. 